\title{
Macrolide antibiotics in diffuse panbronchiolitis and in cystic fibrosis
}

\section{To the Editor:}

We read with interest the editorial by BLACK et al. [1] on the anti-inflammatory effects of macrolide antibiotics. Our interest in this area was stimulated some years ago by reports from Japan which indicated that the introduction of macrolide therapy transformed the prognosis of patients with diffuse panbronchiolitis. This condition, rare outside Asia, affects adults and is characterized by relentless progressive pulmonary damage associated with chonic inflammation. Bronchoalveolar lavage (BAL) reveals high levels of neutrophils within the airways. Frequent infections with Haemophilus influenzae and subsequently chronic Pseudomonas aeruginosa infections are characteristic. The aetiology is unknown.

Traditionally patients were treated with combinations of intravenous and oral antibiotics together with steroid therapy. The introduction of low-dose erythromycin (200 $\mathrm{mg}$ t.d.s.) resulted in a dramatic improvement in the prognosis of these patients with the $10 \mathrm{yr}$ survival of patients colonised with $P$. aeruginosa increasing from 12.4 to greater than $90 \%$ [2]. Newer macrolides have also been shown to have similar beneficial effects. Subsequent studies have indicated that this improvement is accompan- ied by reductions in interleukin (IL)-8, neutrophils and neutrophil elastase levels in BAL [2-5]. The mechanism for this improvement is unclear. As noted by Dr. Black, macrolides have a range of effects on neutrophils and can influence the release and effects of inflammatory products produced by $P$. aeruginosa. Many of these effects were cited by BLACK [1]. Which of these effects play a major role in the reduced levels of inflammation is

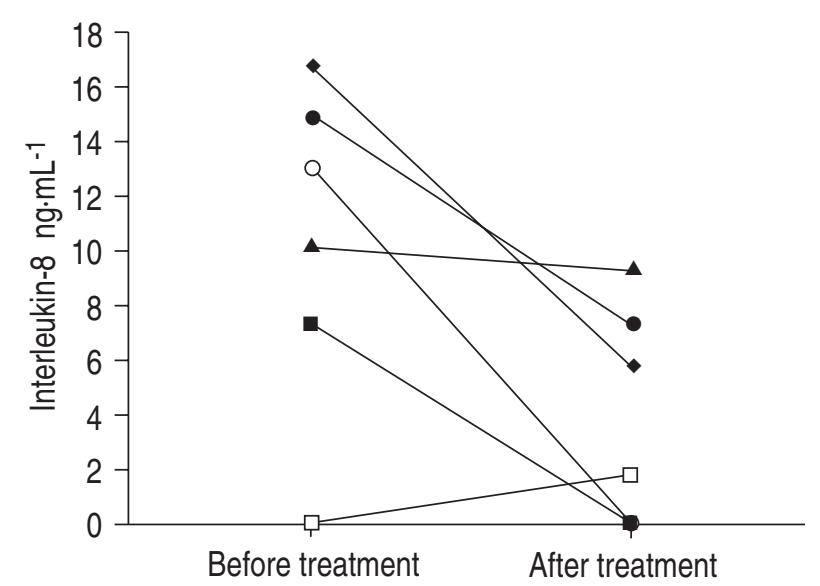

Fig. 1. - The effect of erythromycin (200 mg t.d.s.) on sputum interleukin- 8 in six patients. un-clear though one study has suggested that the benefits of this approach apply equally to patients with or without $P$. aeruginosa infections [2], indicating that the predominant effect may be through reducing neutrophil recruitment.

Stimulated by some of the similarities between the pulmonary inflammation noted in diffuse panbronchiolitis and that characteristic of cystic fibrosis, we undertook an open pilot study in which sputum was obtained from six cystic fibrosis patients at the beginning and end of a 1 month period, during which they were treated with low dose (200 mg t.d.s.) erythromycin. Interestingly, shortly after commencing our pilot study it became clear that others were interested in pursuing this potential anti-inflammatory therapeutic approach [6]. In our study we noted a large reduction in sputum IL-8 levels in four of the six patients with no change in one and, slight increase in the sixth (fig. 1). Neutrophil elastase levels also fell in four of the five paired samples analysed. As a result of these encouraging preliminary results we are now undertaking larger clinical trials and studying the possible mechanisms in more detail in vitro.

\section{References}

1. Black PN. Anti-inflammatory effects of macrolide antibiotics. Eur Respir J 1997; 10: 971-972.

2. Fujii T, Kadota J-I, Iida KK, et al. Long term effect of erythromycin therapy in patients with chronic Pseudomonas aeruginosa infection. Thorax 1995; 50: 12461252.

3. Ichikawa $\mathrm{Y}$, Ninomiya $\mathrm{H}$, Koga $\mathrm{H}$, et al. Erythromycin reduces neutrophils and neutrophil-derived elastolytic-like activity in the lower respiratory tract of bronchiolitis patients. Am Rev Respir Dis 1992; 146: 196-203.

4. Kadota J-I, Sakito O, Kohno S, et al. A mechanism of erythromycin treatment in patients with diffuse panbronchiolitis. Am Rev Respir Dis 1993; 147: 153-159.

5. Oishi K, Sonda F, Kobayashi S, et al. Role of IL-8 and inhibitory effects of erythromycin on IL-8 release in the airways of patients with chronic airways disease. Infect Immun 1994; 62: 4145-4152.

6. Hoiby N. Diffuse panbronchiolitis and cystic fibrosis: East meets West. Thorax 1994; 49: 531-532.

M.L. Everard*, P. Sly ${ }^{+}$, S. Brenan ${ }^{+}$, G. Ryan ${ }^{+}$ *Dept of Paediatric Respiratory Medicine, Sheffield's Children's Hospital UK and +TVWT Institute for Child Health Research, Perth, Western Australia. 